\title{
Extent of Linkage Disequilibrium in Holstein Cattle in North America
}

\author{
M. Sargolzaei, ${ }^{*}$ F. S. Schenkel,, ${ }^{\star 1}$ G. B. Jansen,† and L. R. Schaeffer* \\ ${ }^{*}$ Centre for Genetic Improvement of Livestock, Animal and Poultry Science Department, University of Guelph, Guelph, Ontario, \\ N1G 2W1 Canada \\ †Dekoppel Consulting, Guelph, Ontario, N1G 2Y8 Canada
}

\begin{abstract}
The success of fine-scale mapping and genomic selection depends mainly on the strength of linkage disequilibrium (LD) between markers and causal mutations. With Lewontin's measure of LD (known as $D^{\prime}$ ), high levels of $\mathrm{LD}$ that extend over several million base pairs have been reported in livestock. However, this measure of LD can be strongly biased upward by small samples and by low allele frequencies. The aim of this study was to characterize the level and extent of LD in Holstein cattle in North America (Canada and the United States for purposes of this study) by using the squared correlation of the alleles at 2 loci $\left(r^{2}\right)$. The Affymetrix MegAllele GeneChip Bovine Mapping 10K single nucleotide polymorphism (SNP) array was used to genotype 821 bulls, from which 497 were used in the analysis of the extent of LD. A total of 5,564 SNP were used after filtering out SNP with more than $5 \%$ of Mendelian inconsistencies, with more than $20 \%$ missing genotypes, or with a minor allele frequency of less than $10 \%$. Analysis of syntenic pairs revealed that useful LD (measured as $r^{2}>0.3$ ) occurred at distances shorter than $100 \mathrm{~kb}$. Linkage disequilibrium decayed very rapidly, within a few hundred kilobase pairs. In addition, no substantial LD between unlinked loci was found. Using a sliding window analysis, we observed an irregular pattern of $\mathrm{LD}$ across the genome. These findings suggest that to capture useful LD, which is required for whole-genome fine mapping and genomic selection, a denser SNP map would be needed.
\end{abstract}

Key words: linkage disequilibrium, linkage disequilibrium pattern, Holstein, single nucleotide polymorphism

\section{INTRODUCTION}

Quantifying the level of linkage disequilibrium (LD) or nonrandom association of alleles at different loci is an important step for fine-scale mapping of QTL (e.g.,

Received July 27, 2007.

Accepted December 12, 2007.

${ }^{1}$ Corresponding author: schenkel@uoguelph.ca
Meuwissen and Goddard, 2000), genomic selection (Meuwissen et al., 2001), and increasing the understanding of genomic architecture and the historical population structure (e.g., Hayes et al., 2003). Recent efforts toward construction of a high-density map of single nucleotide polymorphisms (SNP) with low genotyping cost have resulted in a rapid increase in the number of SNP available for genotyping. Consequently, fine mapping of genes with a major effect on quantitative traits has now become more feasible in many livestock species.

Although linkage analysis (e.g., George et al., 2000), which relies on observed pedigree information (i.e., recent genetic relationships) in addition to marker genotypes, can potentially identify wide regions explaining variation for traits of interest, the $\mathrm{LD}$ analysis, which exploits historical recombination events, can be applied as a powerful tool for fine mapping at the population level in unrelated or historically related individuals (Meuwissen and Goddard, 2000). A smaller sample size is needed for LD analysis to achieve the same power, compared with linkage analysis (Risch and Merikangas, 1996).

The success of LD mapping, however, depends greatly on an appropriate balance between the extent of LD and marker density. Measuring LD can help to determine the required marker density in fine mapping. For example, the level of LD in human populations is very low because of large effective population sizes (Kruglyak, 1999), and it is expected that for a whole-genome scan, a dense marker map with resolution of less than $\sim 0.005 \mathrm{cM}(5 \mathrm{~kb})$ is required (Dunning et al., 2000). Conversely, in livestock with smaller effective population sizes, substantial LD is expected to span larger genetic distances (e.g., Farnir et al., 2000; McRae et al., 2002; Odani et al., 2006); therefore, marker maps with less resolution, compared with those of humans, might be required for fine mapping. However, LD is population specific and there is some degree of heterogeneity in the extent of $\mathrm{LD}$ among populations, depending on the heterogeneity of demographic histories (e.g., Laan and Paabo, 1997). In cattle, LD in intensively selected populations is extended over larger distances 
(e.g., Farnir et al., 2000; Tenesa et al., 2003) than LD in moderately selected populations (e.g., Thevenon et al., 2007).

The most commonly used measures of LD are the multiallelic $D^{\prime}$ (Lewontin, 1964), $r^{2}$ (Hill and Robertson, 1968), and standardized $\chi^{2}$ (Yamazaki, 1977; Zhao et al., 2005). The $D^{\prime}$ measure has been commonly used in LD studies in Holstein cattle, in which strong LD over long distances has been reported. However, $D^{\prime}$ is biased upward by small sample sizes and by pairs of markers with low allele frequencies (Ardlie et al., 2002; Du et al., 2007). In addition, there is no clear interpretation for intermediate values of $D^{\prime}$, because the magnitude of $D^{\prime}$ strongly depends on sample size, especially for SNP with rare alleles (Ardlie et al., 2002; Du et al., 2007).

Recently, Zhao et al. (2007) showed that $D^{\prime}$ is strongly inflated compared with $r^{2}$, and they reported high $D^{\prime}$ values even for markers in equilibrium and separated by large distances. Zhao et al. (2007) argued that $r^{2}$ is a more suitable measure for estimating usable $\mathrm{LD}$ between biallelic markers (e.g., SNP). Note that for biallelic markers, standardized $\chi^{2}$ is equivalent to $r^{2}$. Compared with previous studies (e.g., Farnir et al., 2000; Tenesa et al., 2003; Khatkar et al., 2006), recent reports have indicated less LD in dairy cattle based on $r^{2}$ (Goddard et al., 2006; Sandor et al., 2006; Spelman and Coppieters, 2006). The objectives of this study were to quantify the extent of LD by using $r^{2}$, and describe the pattern of $\mathrm{LD}$ across all autosomes in Holstein cattle in North America (Canada and the United States for purposes of this study) by using a 10K SNP array (Affymetrix Inc., 2005).

\section{MATERIALS AND METHODS}

\section{Animals and Gametic Pool}

A set of 821 progeny-tested proven bulls born between 1965 and 2001 was genotyped by using a 10K SNP array (details are discussed in the next section). Bulls comprised 66 sire families with an average family size of 12 sons ( 1 to 55 sons per family). Progeny of sires with family sizes of 5 or less made up only $10 \%$ of all the sampled bulls. The structure of the pedigree was mainly a granddaughter design, but some bulls had a more complex general pedigree structure going back 5 generations among the genotyped animals. All of the 821 bulls sampled have genetically contributed to the current Canadian cow population.

The 821 genotyped bulls were used for haplotype reconstruction (see the section on haplotyping). From this sample, 497 bulls were selected, and from these bulls maternal haplotypes were used to evaluate the extent and pattern of LD. Maternal haplotypes were used, because LD from paternal haplotypes may reflect LD within sire families, rather than in the wider population of Holstein cattle in North America. Maternal gametes have also been used with similar population structures by other researchers (Farnir et al., 2000; Odani et al., 2006; Spelman and Coppieters, 2006).

The 497 selected bulls were all from dams born in North America and from sire families with $\geq 8$ sibs. Bulls were also selected for not being maternal half-sibs, and were all born after 1990. Of the 497 dams, 303 and 194 dams were born in Canada and the United States, respectively. The sampled dams were representative of the Holstein population in North America because of the extensive exchange of genetic materials between Canada and the United States. After tracing the pedigree of 497 selected bulls to 1910 , the genetic contributions of founders (animals with unknown parents) from the United States to the 303 dams from Canada and to the 194 dams from the United States were 59 and $70 \%$, respectively.

\section{Genotypes}

The DNA samples were extracted from semen samples, and genotyping was carried out by using the Affymetrix MegAllele GeneChip Bovine Mapping 10K SNP array (Affymetrix Inc., 2005). Of the 821 bulls, 428 bulls were genotyped at Affymetrix Inc. (South San Francisco, CA) and 393 bulls were genotyped at the Center for Applied Genomics of the Hospital for Sick Children (Toronto, Canada). To verify the consistency of genotyping between the 2 laboratories, 10 bulls were genotyped in both laboratories. The frequency of disagreements between the SNP genotypes from the 2 laboratories for the 10 bulls (excluding failed SNP) was less than 1\%, which showed a very high level of agreement between the 2 sets of genotypes. Of the 9,919 SNP genotyped, 192 SNP failed to produce genotypes for all bulls. With the bovine sequence assembly Btau 3.1 obtained from the International Bovine Genome Sequencing Consortium (ftp://ftp.hgsc.bcm.tmc.edu/pub/data/Btaurus/ fasta/Btau20060815-freeze/), 8,624 SNP had known physical locations (in base pairs) on the 29 Bos taurus autosomal chromosomes (BTA).

Genotyping errors can substantially influence LD measures. Therefore, in LD studies, it is important to minimize possible genotyping errors (Akey et al., 2001). The SNP were checked for Mendelian segregation inconsistencies (attributed to genotyping errors) and SNP with more than 5\% genotyping errors were removed from the analysis. For the remaining SNP, eventual Mendelian inconsistencies were resolved by using an adapted version of the Genotype Elimination algorithm (Lange and Goradia, 1987). Single nucleotide polymor- 
phisms with more than $20 \%$ missing genotypes or with minor allele frequencies (MAF) of less than $10 \%$ were filtered out. After editing, a total of 5,564 SNP were used in the analyses.

\section{Haplotyping}

In general, the haplotype phase is not known but it can be inferred from within-family or population-wide information. In selected populations, haplotypes are usually inferred based on family information. In small or relatively small sample sizes, the use of family information can help to resolve the haplotypes, depending on family size. However, haplotype reconstruction from observed genotyping data within a pedigree is a challenging problem when there are many markers. For a large number of markers, the number of possible haplotypes is usually too big to handle, because the number of haplotypes quickly increases as the number of markers increases. In such a situation, most methods are not guaranteed to find the most probable haplotype (Gao et al., 2004).

Paternal and maternal haplotypes were reconstructed by using a rapid algorithm developed specifically to handle large numbers of biallelic markers in a general pedigree of paternal half-sib families. In the haplotype reconstruction, all 821 genotyped bulls were considered. The algorithm used is described in detail in Appendix 1. Only males were assumed to be genotyped and all dams were assumed to be unrelated. The probability, $P_{i j}$, that animal $i$ inherited allele 1 from its sire at locus $j$ was approximated at every locus. Initially, $P_{i j}$ was set to 0 or 1 , as appropriate, for homozygous loci and for heterozygous loci at which the sire is homozygous, and to 0.5 for all remaining heterozygous loci. If the sire had not been genotyped, the probability was arbitrarily set to 1 at the first heterozygous locus on each chromosome. The pedigree was processed twice from youngest to oldest in each of the iterations, first adjusting $P_{i j}$ for information on progeny genotypes at the locus in question and at a nearby informative marker, and second readjusting $P_{i j}$ for information on nearby flanking markers for which the sire was heterozygous. Convergence was monitored as the sum of squared changes in $P_{i j}$. Finally, the paternal allele at each heterozygous locus was set to 1 for $P_{i j} \leq 0.5$ and to 2 for $P_{i j}>0.5$.

The algorithm was tested by Monte Carlo simulation by using the pedigree structure of the 821 genotyped bulls. Maternal gametes and gametes from sires not among the 821 genotyped bulls were generated assuming a random mating base population in complete linkage equilibrium. Ten chromosomes of length 10 or 100 cM were simulated, each containing $11,21,51,101$,
201, or 1,001 equally spaced biallelic markers with MAF 0.2 . The simulation was replicated 10 times for each scenario and the mean and standard deviation of the fraction of correctly determined paternal alleles at all heterozygous loci was computed across the 789 animals with a genotyped sire.

\section{Measure of Linkage Disequilibrium}

Linkage disequilibrium was measured in the maternal gametic pool by using $r^{2}$, which is the squared correlation of the alleles at 2 loci (Hill and Robertson, 1968). The $r^{2}$ is the preferred measure of $\mathrm{LD}$ for biallelic markers (e.g., Zhao et al., 2005; Du et al., 2007) and is the most suitable measure for estimating usable LD between SNP (Zhao et al., 2007). The $r^{2}$ was calculated as follows:

$$
r^{2}=\frac{D^{2}}{f(A) f(a) f(B) f(b)},
$$

where $D=f(A B)-f(A) f(B)$, and $f(A B), f(A), f(a), f(B)$, and $f(b)$ are observed frequencies of haplotype $A B$ and of alleles $A, a, B$, and $b$, respectively.

Although $r^{2}$ takes into account differences in allelic frequencies, no measure of LD is completely allele frequency independent (Lewontin, 1988), but many studies have shown that, unlike $D^{\prime}, r^{2}$ is not as sensitive to allele frequencies (e.g., Du et al., 2007; Zhao et al., 2007). In the current investigation, the effect of allele frequency on $r^{2}$ across different distances was also assessed. For all possible SNP pairs, maternal allele and haplotype frequencies were obtained by allele and haplotype counting, and only pairs for which both loci have MAF equal to or greater than $10 \%$ were examined.

\section{Within-Chromosomal and Interchromosomal Heterogeneities}

A general linear model analysis of covariance was conducted to examine within-chromosomal and interchromosomal heterogeneities in LD. Chromosomes and log-transformed genetic distances were fit by using the following linear model:

$$
r_{i j}^{2}=\mu+C_{i}+\beta\left(\log \left(d_{j}\right)-\log (\bar{d})\right)+e_{i j},
$$

where $r_{i j}^{2}$ is the LD between the $j$ th pair of SNP on chromosome $i, \mu$ is the overall mean of $r_{i j}^{2}$ across all syntenic SNP pairs, $C_{i}$ is the mean effect of chromosome $i, d_{j}$ is the genetic distance (in cM) between the $j$ th pair of SNP, $\bar{d}$ is the average genetic distance between all possible syntenic SNP pairs, $\beta$ is the regression coeffi- 
Table 1. Summary of analyzed single nucleotide polymorphism (SNP) markers for each Bos taurus autosome (BTA)

\begin{tabular}{|c|c|c|c|c|c|c|}
\hline BTA & $\begin{array}{l}\text { Length } \\
(\mathrm{Mb})\end{array}$ & $\begin{array}{l}\text { SNP } \\
(\mathrm{n})\end{array}$ & $\begin{array}{c}\text { Mean } \\
\text { distance } \pm \mathrm{SD}^{1} \\
(\mathrm{Mb})\end{array}$ & $\begin{array}{l}\text { Longest gap }{ }^{2} \\
(\mathrm{Mb})\end{array}$ & $\underset{(\mathrm{n})}{\text { Gaps }}>2 \mathrm{Mb}^{1}$ & $\begin{array}{c}\text { Mean } \\
r^{2} \pm \mathrm{SD}^{1}\end{array}$ \\
\hline 1 & 145.88 & 328 & $0.44 \pm 0.69$ & 5.21 & 11 & $0.32 \pm 0.38$ \\
\hline 2 & 125.27 & 281 & $0.45 \pm 0.62$ & 4.02 & 6 & $0.31 \pm 0.36$ \\
\hline 3 & 116.29 & 299 & $0.39 \pm 0.51$ & 3.22 & 4 & $0.27 \pm 0.34$ \\
\hline 4 & 110.79 & 264 & $0.41 \pm 0.59$ & 3.10 & 8 & $0.31 \pm 0.37$ \\
\hline 5 & 118.14 & 259 & $0.45 \pm 0.63$ & 4.27 & 13 & $0.33 \pm 0.36$ \\
\hline 6 & 111.65 & 253 & $0.43 \pm 0.54$ & 3.31 & 6 & $0.28 \pm 0.34$ \\
\hline 7 & 100.74 & 218 & $0.45 \pm 0.62$ & 3.49 & 6 & $0.29 \pm 0.32$ \\
\hline 8 & 103.23 & 257 & $0.40 \pm 0.52$ & 2.93 & 7 & $0.27 \pm 0.34$ \\
\hline 9 & 94.57 & 188 & $0.49 \pm 0.67$ & 2.94 & 13 & $0.31 \pm 0.38$ \\
\hline 10 & 95.75 & 223 & $0.43 \pm 0.59$ & 4.39 & 4 & $0.34 \pm 0.35$ \\
\hline 11 & 100.32 & 243 & $0.41 \pm 0.48$ & 2.22 & 3 & $0.29 \pm 0.34$ \\
\hline 12 & 77.41 & 187 & $0.41 \pm 0.60$ & 2.95 & 6 & $0.31 \pm 0.36$ \\
\hline 13 & 82.75 & 232 & $0.35 \pm 0.54$ & 3.06 & 6 & $0.40 \pm 0.39$ \\
\hline 14 & 81.90 & 220 & $0.37 \pm 0.54$ & 2.89 & 7 & $0.35 \pm 0.37$ \\
\hline 15 & 74.72 & 187 & $0.39 \pm 0.55$ & 2.75 & 6 & $0.31 \pm 0.38$ \\
\hline 16 & 71.89 & 183 & $0.39 \pm 0.55$ & 2.81 & 4 & $0.34 \pm 0.37$ \\
\hline 17 & 69.54 & 167 & $0.42 \pm 0.53$ & 2.46 & 4 & $0.28 \pm 0.34$ \\
\hline 18 & 62.24 & 164 & $0.37 \pm 0.60$ & 3.48 & 6 & $0.29 \pm 0.37$ \\
\hline 19 & 62.70 & 133 & $0.47 \pm 0.63$ & 3.07 & 4 & $0.25 \pm 0.30$ \\
\hline 20 & 67.33 & 162 & $0.42 \pm 0.56$ & 2.47 & 5 & $0.33 \pm 0.38$ \\
\hline 21 & 62.91 & 117 & $0.54 \pm 0.70$ & 4.34 & 6 & $0.22 \pm 0.31$ \\
\hline 22 & 59.02 & 164 & $0.35 \pm 0.54$ & 2.94 & 4 & $0.35 \pm 0.38$ \\
\hline 23 & 48.27 & 135 & $0.35 \pm 0.48$ & 3.06 & 1 & $0.30 \pm 0.35$ \\
\hline 24 & 60.03 & 144 & $0.42 \pm 0.50$ & 1.98 & 0 & $0.28 \pm 0.37$ \\
\hline 25 & 41.93 & 108 & $0.38 \pm 0.43$ & 1.89 & 0 & $0.25 \pm 0.33$ \\
\hline 26 & 47.50 & 126 & $0.37 \pm 0.50$ & 2.52 & 2 & $0.33 \pm 0.37$ \\
\hline 27 & 43.22 & 99 & $0.44 \pm 0.65$ & 3.94 & 2 & $0.33 \pm 0.37$ \\
\hline 28 & 37.68 & 124 & $0.31 \pm 0.47$ & 3.15 & 1 & $0.29 \pm 0.35$ \\
\hline 29 & 44.88 & 99 & $0.46 \pm 0.65$ & 3.65 & 2 & $0.31 \pm 0.38$ \\
\hline Overall & $2,318.56$ & 5,564 & $0.41 \pm 0.58^{2}$ & $3.19^{2}$ & $5.1^{2}$ & $0.31 \pm 0.36^{2}$ \\
\hline
\end{tabular}

${ }^{1}$ Between adjacent SNP.

${ }^{2}$ Overall mean.

cient of LD extent on the log-transformed genetic distance, and $e_{i j}$ is a residual effect. The relationship between physical distance and LD is not monotonic. Therefore, $\log _{10}$-transformed genetic distance was used in lieu of physical distance because of its linear relationship with LD (McRae et al., 2002).

Physical distances were transformed into genetic distance by using the microsatellite marker map from the National Center for Biotechnology Information (2007), for which both physical and genetic maps were available. The physical positions of SNP located within each microsatellite bracket were linearly interpolated to a genetic distance by using the known genetic map of microsatellite markers. The average resolution of the National Center for Biotechnology Information map was $3.93 \mathrm{cM}$, with $3.6 \mathrm{cM}$ standard deviation.

\section{Pattern of LD Along Chromosomes}

The pattern of pairwise measures of $r^{2}$ over the BTA was examined by using a sliding window approach (Zhao et al., 2003). The SNP were not evenly spaced across the genome and tended to be clustered in some regions. Thus, to partially account for SNP density, SNP spaced between 0.2 and $0.6 \mathrm{Mb}$ were considered. A window spanning $2 \mathrm{Mb}$ was moved along each chromosome, with a step size of $0.2 \mathrm{Mb}$. The mean $r^{2}$ in each window was calculated if at least 6 SNP pairs were present. The window size and SNP spacing were chosen based on the SNP map density available.

\section{Effective Population Size}

As additional information, the effective population size was determined based on $r^{2}$ measures. Following Sved (1971), and assuming a model without mutation, the effective population size $\left(N_{e}\right)$ can be determined by using the following expectation for $r^{2}$ :

$$
E\left(r^{2}\right)=\frac{1}{1+4 N_{e} c},
$$

where $c$ is the recombination distance (in morgans) between the SNP. Because of the relatively large sample 
size in this study, the effect of sample size was assumed to be negligible. To account for recombination variation across the genome, the interpolated genetic distance in morgans, as described previously, was used. Because LD breaks down more rapidly over generations for loci further apart, LD at large distances reflects $N_{e}$ at recent generations. The age of $N_{e}$ for any distance can be roughly estimated by $1 /(2 c)$ (Hayes et al., 2003). Hence, $N_{e}$ in earlier generations was estimated at different time points, back to 500 generations, by taking the mean LD for a range of distances. The SNP pairs were sorted according to the distances between them, and then the mean LD was obtained at every $0.1( \pm 0.05)$, $0.5( \pm 0.5)$, and $1( \pm 1) \mathrm{cM}$ for distance ranges of 0.1 to 1,1 to 10 , and 10 to $20 \mathrm{cM}$, respectively. The main force that breaks down LD is recombination, and because recombination is rare between SNP separated by short distances, a small interval was considered for these distances to increase the accuracy of estimation of $N_{e}$ in the remote past.

\section{RESULTS}

A descriptive summary of chromosomes and SNP is shown in Table 1. The total autosomal genome length was 2,318.56 Mb, with the shortest BTA being 37.68 $\mathrm{Mb}$ and the longest BTA being 145.88 Mb. The density of the SNP loci varied among the chromosomes and ranged from 3.29 SNP/Mb in BTA 28 to $1.86 \mathrm{SNP} / \mathrm{Mb}$ in BTA 21. However, SNP were not uniformly distributed across the autosomes and tended to be clustered in some regions. The largest gap was observed on BTA 1 (5.21 Mb).

Figure 1 presents the distribution of MAF in the set of 497 maternal haplotypes. More than $84 \%$ of SNP had MAF larger than 0.2 , implying that the effect of low MAF on the overall LD estimates should be small.

The mean percentages (SD) of paternal alleles incorrectly assigned at all heterozygous loci in the simulated data sets by the rapid algorithm used for haplotype reconstruction were 8.67 (0.54), 2.83 (0.24), 0.86 (0.09), and $0.62(0.05)$ for $11,21,51$, and $101 \mathrm{SNP}$, respectively, on a 10 -cM chromosomal segment and $4.53(0.21), 2.46$ (0.09), 1.43 (0.04), and $0.62(0.04)$ for $51,101,201$, and 1,001 SNP, respectively, on a 100-cM chromosome. By trial and error, the percentage of correct calls was found to be maximized by performing approximately 10 iterations of the algorithm, even though the convergence criteria continued to decline beyond 100 iterations. The program for haplotype reconstruction completed 10 iterations for the real data set in less than a minute on an off-the-shelf laptop computer. Considering the average number of markers per chromosome and the unequal spacing of the markers in the real data, the

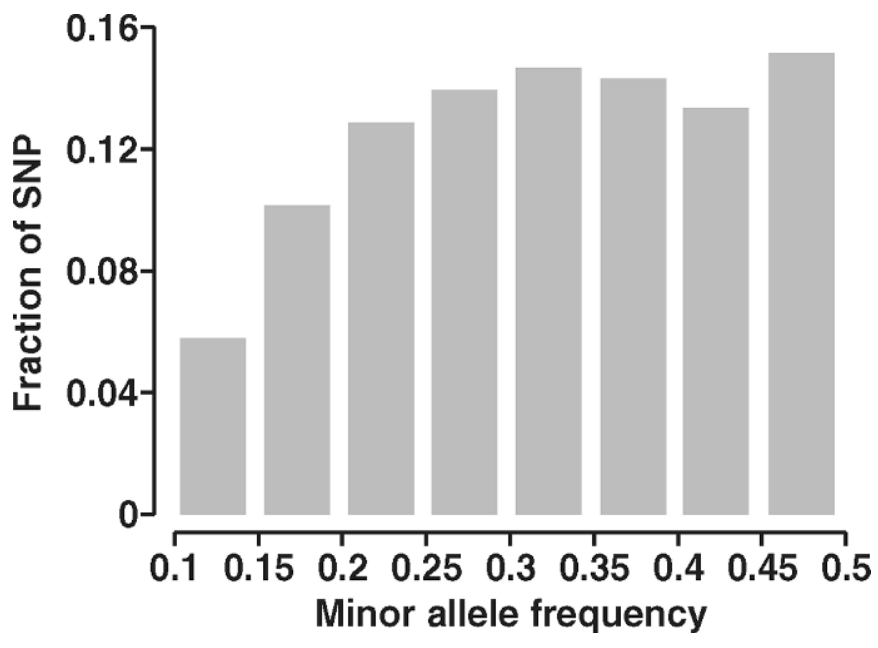

Figure 1. Distribution of single nucleotide polymorphisms (SNP) over minor allele frequency in maternal haplotypes.

percentage of incorrect phase calls could be expected to be approximately $2 \%$.

The mean $r^{2}$ (SD) between adjacent SNP ranged from $0.22(0.31)$ for BTA 21 to $0.40(0.39)$ for BTA 13, and the overall $r^{2}$ was $0.31(0.36)$. Figure 2 depicts the distribution of distances between adjacent SNP pairs. Fortyone percent of the SNP pairs (2,294 pairs) were less than $0.1 \mathrm{Mb}$ apart and only $13 \%$ of pairs (734 pairs)

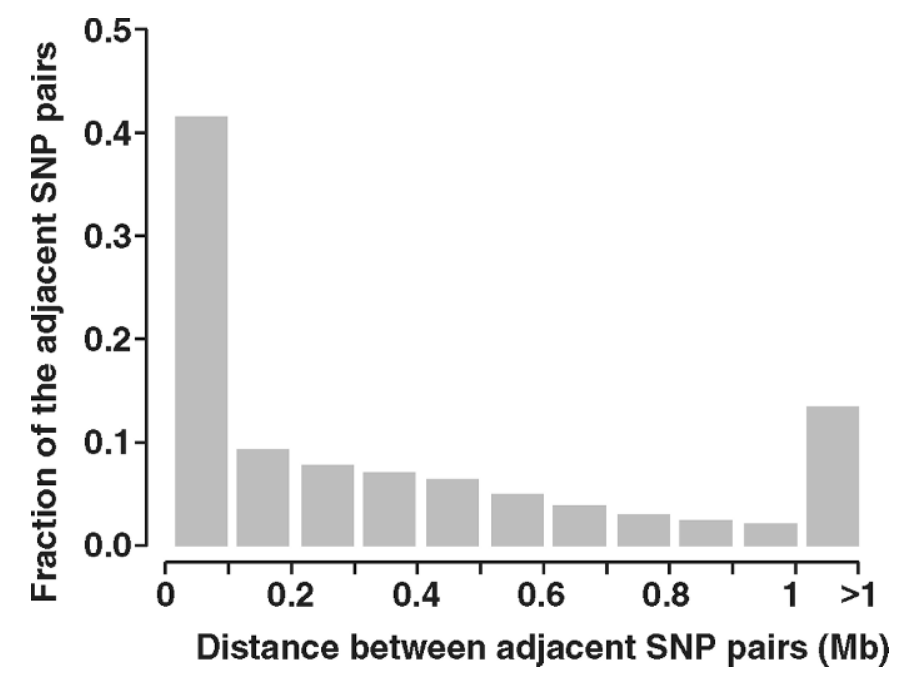

Figure 2. Distribution of number of adjacent single nucleotide polymorphism (SNP) pairs according to their physical distance. 


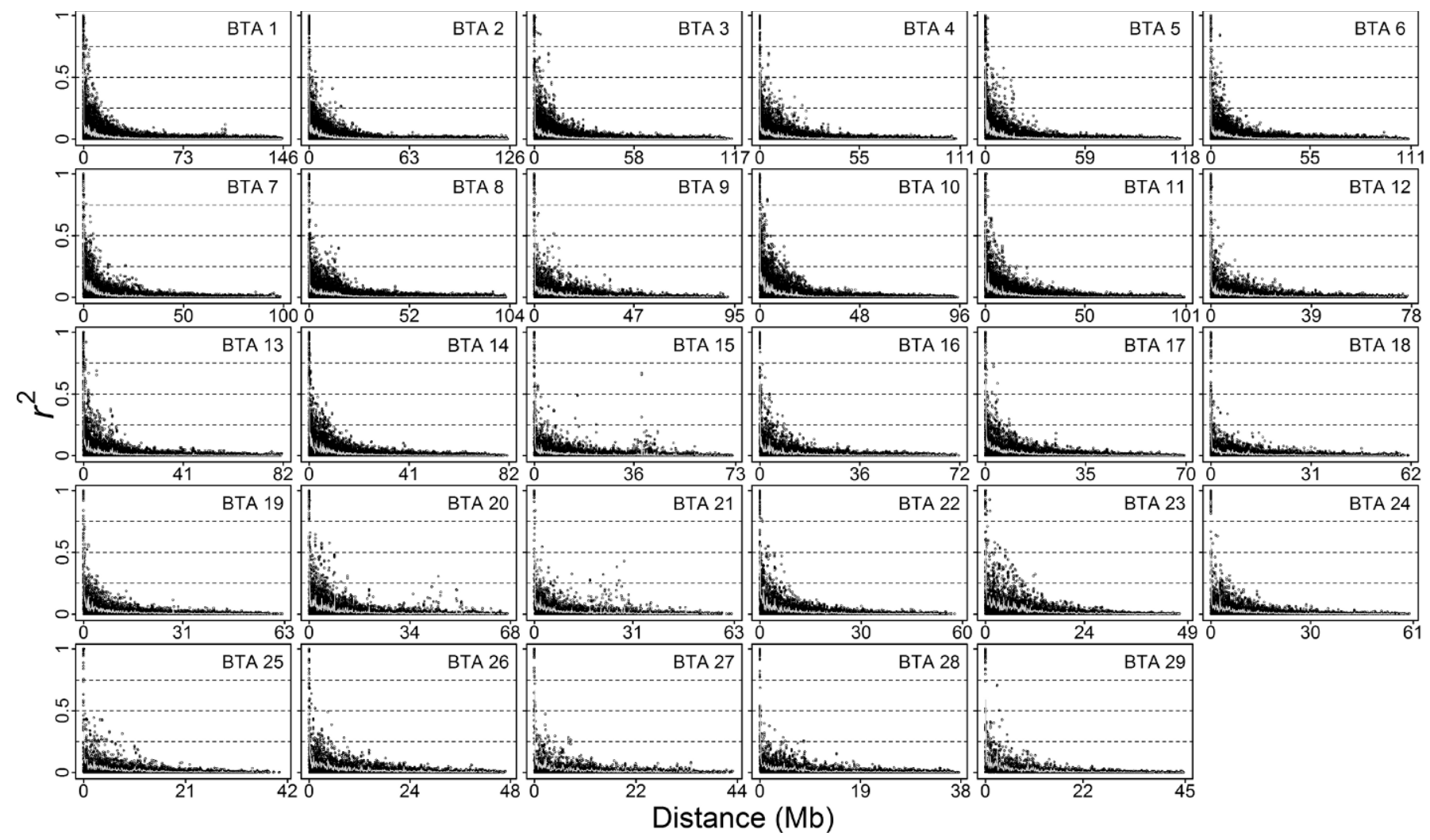

Figure 3. Distribution of $r^{2}$ between syntenic single nucleotide polymorphism (SNP) pairs as a function of physical distance. The mean $r^{2}$ over successive intervals of $0.1 \mathrm{Mb}$ is plotted in gray.

were more than $1 \mathrm{Mb}$ apart, indicating a relatively dense SNP map. The mean distance, median, and standard deviation were $0.41,0.19$, and $0.58 \mathrm{Mb}$, respectively.

Level of LD decay, as a function of distance between pairs of SNP, is shown for each chromosome in Figure 3. Greater LD were observed only at small distances between pairs of SNP. Table 2 presents the mean $r^{2}$ for different distances (0.1-Mb intervals) between SNP

Table 2. Frequency and mean $r^{2}\left(\bar{r}^{2}\right)$ for different distances between closely located syntenic SNP pairs

\begin{tabular}{lrcrc}
\hline $\begin{array}{l}\text { Distance } \\
\text { range }(\mathrm{Mb})\end{array}$ & Pairs (n) & \multicolumn{1}{c}{$\bar{r}^{2} \pm \mathrm{SD}$} & $\begin{array}{r}\text { Frequency } \bar{r}^{2}>0.3 \\
(\%)\end{array}$ \\
\hline $0.0-0.1$ & 4,135 & $0.58 \pm 0.37$ & $2,826(68.34)$ \\
$0.1-0.2$ & 1,343 & $0.15 \pm 0.19$ & $226(16.83)$ \\
$0.2-0.3$ & 1,394 & $0.11 \pm 0.15$ & $170(12.20)$ \\
$0.3-0.4$ & 1,414 & $0.10 \pm 0.13$ & $99(7.00)$ \\
$0.4-0.5$ & 1,298 & $0.09 \pm 0.13$ & $85(6.55)$ \\
$0.5-0.6$ & 1,414 & $0.09 \pm 0.12$ & 91 & $(6.44)$ \\
$0.6-0.7$ & 1,308 & $0.09 \pm 0.11$ & 65 & $(4.97)$ \\
$0.7-0.8$ & 1,223 & $0.08 \pm 0.12$ & 77 & $(6.30)$ \\
$0.8-0.9$ & 1,233 & $0.08 \pm 0.10$ & $63(5.11)$ \\
$0.9-1.0$ & 1,279 & $0.08 \pm 0.10$ & 56 & $(4.38)$ \\
Overall & 580,517 & $0.02 \pm 0.07$ & 5,204 & $(0.90)$ \\
\hline
\end{tabular}

located less than $1 \mathrm{Mb}$ apart. High $\mathrm{LD}$ values were observed for SNP located in close proximity. The mean $r^{2}$ for pairs of SNP closer than $0.1 \mathrm{Mb}$ was 0.58 , and $68.3 \%$ of these pairs had $r^{2}$ larger than 0.3 . Disequilibrium decreased sharply with increasing distance between SNP.

Decay of LD showed a clear exponential trend with physical distance. At ranges 0 to $0.1,0$ to 0.2 , and 0 to $0.5 \mathrm{Mb}, 68.3,55.7$, and $35.5 \%$ of SNP pairs exhibited $r^{2}$ larger than 0.3 , respectively. A total of 491 pairs of linked SNP were in complete LD $\left(r^{2}=1\right)$. In general, there was more variation among the $r^{2}$ values the closer were the SNP pairs, especially for SNP closer than 0.1 $\mathrm{Mb}$ (Figure 3 and Table 2).

Linkage disequilibrium levels in gametic samples from dams born in Canada and the United States were also assessed separately. However, very similar LD statistics for both groups were found (Table 3). Because in different populations or breeds a pair of SNP may have the same value of $r^{2}$ but different LD phases (Goddard et al., 2006), the persistency of the LD phase between SNP in the 2 groups was also assessed. This was done by computing the regression of signed $r$ in one group on signed $r$ in another group for the same SNP 
Table 3. Mean $\bar{r}^{2}$ and regression coefficients $(\beta)$ of the linear regression of $r$ in the maternal gametic pool from Canada on $r$ in the maternal gametic pool from the United States for a range of distances

\begin{tabular}{lccc}
\hline \multirow{2}{*}{$\begin{array}{l}\text { Distance } \\
\text { range }(\mathrm{Mb})\end{array}$} & Mean $\bar{r}^{2} \pm \mathrm{SD}$ & \\
\cline { 2 - 3 } & Canada & United States & $\beta$ \\
\hline $0.0-0.1$ & $0.59 \pm 0.37$ & $0.59 \pm 0.37$ & 0.99 \\
$0.1-0.2$ & $0.16 \pm 0.19$ & $0.16 \pm 0.20$ & 0.99 \\
$0.2-0.3$ & $0.12 \pm 0.15$ & $0.12 \pm 0.16$ & 0.98 \\
$0.3-0.4$ & $0.11 \pm 0.14$ & $0.11 \pm 0.13$ & 1.00 \\
$0.4-0.5$ & $0.10 \pm 0.15$ & $0.10 \pm 0.13$ & 0.99 \\
$0.5-0.6$ & $0.10 \pm 0.12$ & $0.09 \pm 0.12$ & 0.97 \\
$0.6-0.7$ & $0.09 \pm 0.12$ & $0.09 \pm 0.12$ & 1.00 \\
$0.7-0.8$ & $0.09 \pm 0.13$ & $0.09 \pm 0.13$ & 0.99 \\
$0.8-0.9$ & $0.08 \pm 0.11$ & $0.08 \pm 0.11$ & 1.01 \\
$0.9-1.0$ & $0.08 \pm 0.11$ & $0.08 \pm 0.10$ & 0.99 \\
$1.0-10.0$ & $0.05 \pm 0.07$ & $0.05 \pm 0.07$ & 0.99 \\
\hline
\end{tabular}

pairs for different distance ranges ( 0 to $10 \mathrm{Mb}$ ). Regression coefficients for all distance ranges were very close to one (Table 3). Therefore, the results strongly suggest that the sampled maternal haplotypes are representative of one population.

To assess the effect of allele frequencies on $r^{2}$, MAF for both loci were divided into 8 groups (with an increment in frequency of 5\%), and the mean $r^{2}$ and frequency of SNP pairs for every possible combination of MAF groups in locus 1 and MAF groups in locus 2 was then obtained. The mean $r^{2}$ and frequency of SNP pairs were plotted against MAF groups at both loci in 2 dimensions for different distances between SNP. Results for SNP pairs located nearer than $150 \mathrm{~kb}$ and SNP pairs located between 150 - and $300-\mathrm{kb}$ distances are shown in Figure 4. For very small distances, mean pairwise $r^{2}$ increased as the difference in MAF decreased. Although for SNP pairs separated by $\leq 150 \mathrm{~kb}$, MAF seemed to have a large effect on the mean $r^{2}$, the larger proportion of SNP pairs in categories with the same allele frequencies indicated that useful LD might be only slightly underestimated. In this study, the useful level of $\mathrm{LD}$ was defined to be $r^{2}>0.3$ (Ardlie et al., 2002). Generally, small proportions of SNP pairs with very similar MAF were observed for distances longer than $0.15 \mathrm{Mb}$. In all distance ranges, usually a lower mean $r^{2}$ was observed when the difference in MAF was larger.

Covariance analysis of the $r^{2}$ for all syntenic SNP pairs revealed a significant linear effect of the $\log _{10^{-}}$ transformed distance, as well as a significant effect of the chromosome $(P<0.0001$ in both cases). However, as expected, most of the variation in $r^{2}$ was explained by the $\log _{10}$ distance (the correlation between $r^{2}$ and the $\log _{10}$-transformed genetic distance ranged from -0.74 to -0.58 , depending on the BTA).

The LD for all possible nonsyntenic SNP pairs was also assessed. The mean $r^{2}$ (SD) was $0.003(0.004)$ for

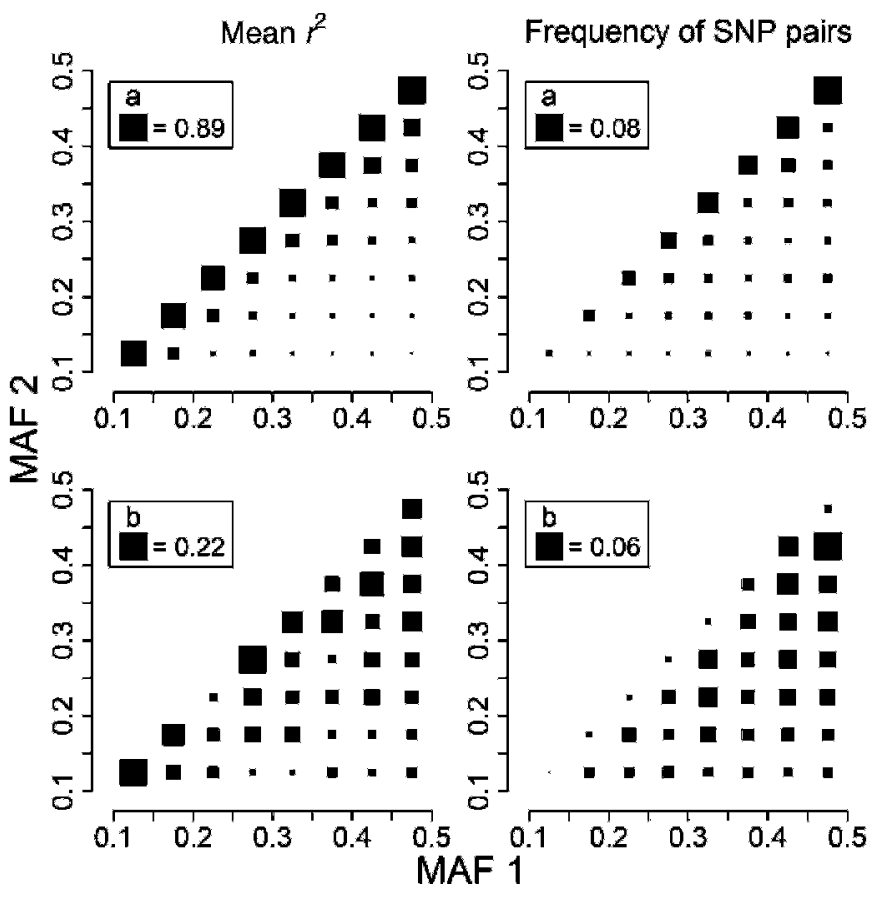

Figure 4. Mean $r^{2}$ and frequency of single nucleotide polymorphism (SNP) pairs across minor allele frequency (MAF) ranges for SNP pairs located $\leq 150 \mathrm{~kb}$ apart (a) and between 150 and $300 \mathrm{~kb}$ apart (b). Both MAF were sorted in 8 bins of size 0.05 (from 0.1 to 0.5 ). The size of square represents the magnitude of mean $r^{2}$ or frequency of SNP pairs for each category relative to the maximum value given in the figure.

$14,717,678$ nonsyntenic SNP pairs. The $r^{2}$ for $99.9 \%$ of the nonsyntenic SNP pairs were lower than 0.04. Only 10 nonsyntenic SNP pairs showed $r^{2}$ of greater than 0.3 .

Regions with low and high levels of $\mathrm{LD}$ based on the sliding window approach (Figure 5) were also examined. The mean $r^{2}$ could not be obtained for many regions because of the insufficient number of well-spaced SNP in those regions. Only $44.5 \%$ of the autosomal genome was assessed. Occasional long-range LD was observed, and there was a substantial level of variation in patterns of $\mathrm{LD}$ across the genome. Out of 2,597 examined windows, 3.6 and $64.5 \%$ had a mean $r^{2}>0.3$ and $r^{2}<0.1$, respectively. Large blocks with a high level of LD $\left(\bar{r}^{2}>0.3\right)$ were not found for 16 out of 29 BTA.

Following Hayes et al. (2003), $N_{e}$ for different times during the past were estimated (Figure 6 ). The recent $N_{e}$ and ancestral $N_{e}$ at 250 and 500 generations ago were less than $100, \sim 1,000$, and $\sim 1,400$ individuals, respectively, showing clear evidence of a decline in effective population size.

\section{DISCUSSION}

Animal genetics research has undergone a significant change over the last decade with increasing interest in 


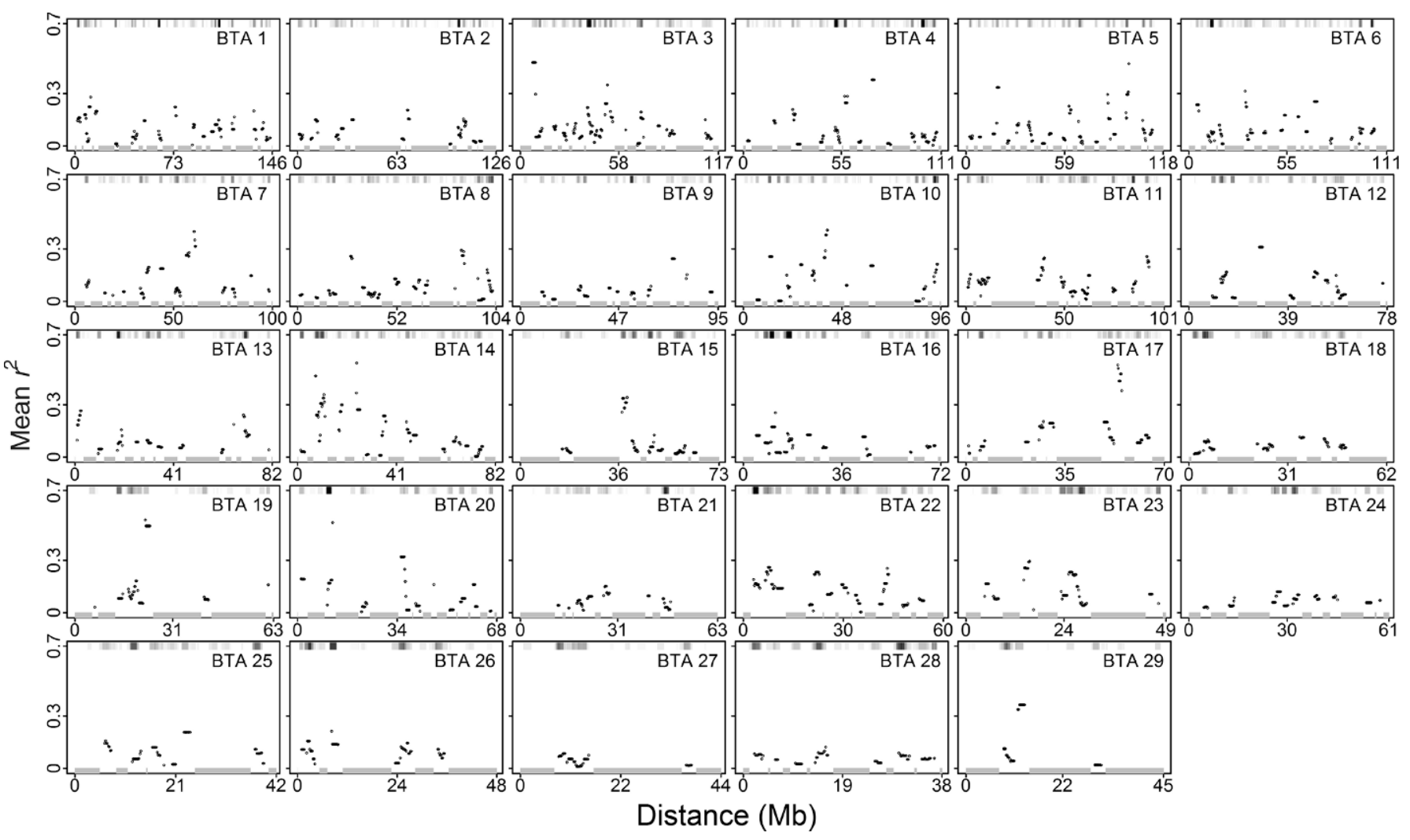

Figure 5. Mean $r^{2}$ based on the sliding window approach. The top axis represents the number of single nucleotide polymorphism (SNP) pairs evaluated in each window in different shades. The darker the line, the greater the number (the darkest indicator corresponds to the number of SNP pairs $\geq 36$ ). The gray strip at the bottom axis shows regions that were not covered by any window.

genomics. Intense efforts have been made to uncover the genetic architecture, detect causal mutations, and make use of markers in selection schemes based on the presence of LD. Knowledge of the extent of LD is critical for designing LD mapping experiments and to interpret the results of these experiments. Several studies have suggested that high levels of LD are extended over long distances in dairy cattle (e.g., Farnir et al., 2000; Tenesa et al., 2003; Khatkar et al., 2006). In contrast, from the results of a few recent studies, the level of $\mathrm{LD}$ in cattle populations might be lower than previously thought (Goddard et al., 2006; Sandor et al., 2006; Spelman and Coppieters, 2006). The current study examined a large number of SNP to measure the extent of LD in Holstein cattle in North America. Results revealed that the useful level of LD extended over short distances, yet it extended over much longer distances compared with LD in humans. For SNP pairs less than $100 \mathrm{~kb}$ apart, substantial LD $\left(\bar{r}^{2}=0.58\right)$ was found. More precisely, mean $r^{2}$ was 0.73 for SNP separated by $10 \mathrm{~kb}$, which was very similar to that reported for Holstein cattle in Australia (Goddard et al., 2006). In general, the mean LD declined rapidly with increasing distance between
SNP to a very low level (Figure 3). The decline in mean LD was an exponential function of distance, being very small for distances larger than $20 \mathrm{Mb}$. However, it is difficult to compare the level and extent of $L D$ obtained in different studies because of different sample sizes, LD measures, marker types, marker densities, and recent and historical population demographics (Pritchard and Przeworski, 2001). Each of these factors can affect the estimates of LD. For example, with the $D^{\prime}$ measure, a high level of LD extending over more than $10 \mathrm{cM}$ has been reported (e.g., Farnir et al., 2000; Khatkar et al., 2006; Odani et al., 2006), but this measure can be inflated at large distances, for SNP with low allele frequencies, and by small sample sizes (Zhao et al., 2005; Du et al., 2007; Zhao et al., 2007).

To assess the robustness of $r^{2}$, its dependency on allele frequencies was investigated. Variation in $r^{2}$ across different ranges of MAF was high for small distances. The $r^{2}$ tended to be substantially larger at very close SNP pairs ( $\leq 150 \mathrm{~kb}$ ) when allele frequencies were similar (Figure 4). Uimari et al. (2005) also reported that $r^{2}$ was dependent on allele frequencies at very short distances. In another study, Du et al. (2007) 


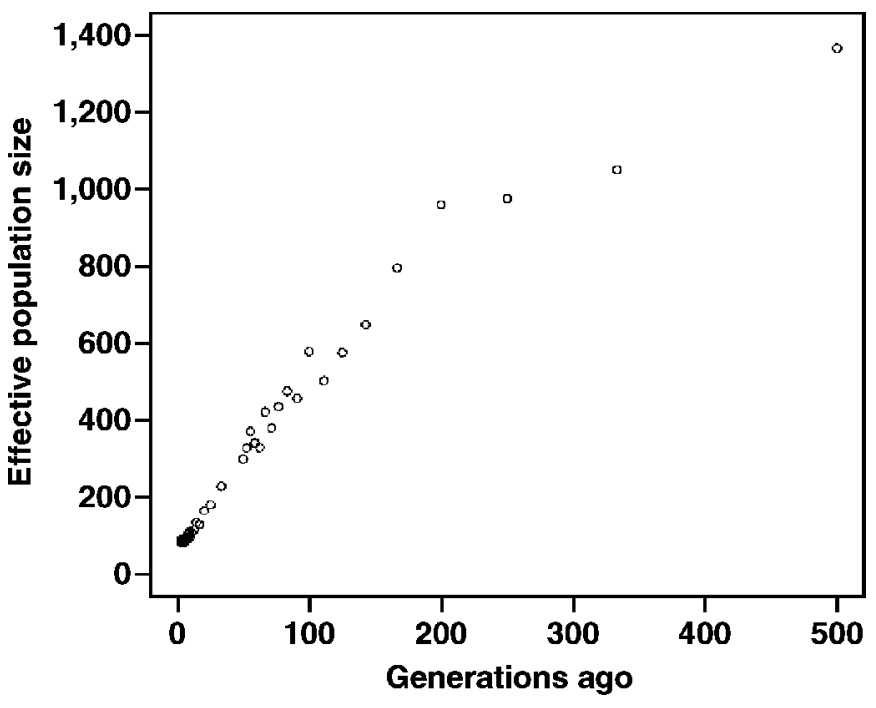

Figure 6. Estimated historical effective population size over time.

showed that $r^{2}$ was inflated when the mean MAF was either too low $(\sim 0)$ or too high $(\sim 0.5)$. However, these authors did not report the distribution of SNP pairs across different levels of MAF; thus, their results cannot be directly compared with the results obtained here.

In the current study, at short distances $(\leq 150 \mathrm{~kb})$, the proportions of SNP pairs in groups with similar ranges of MAF were quite large compared with those in groups with different ranges of MAF (Figure 4). However, at longer distances $(>150 \mathrm{~kb})$ the inverse relationship was observed. In fact, when there was LD between the 2 loci, the allele frequencies of the 2 loci were correlated and the degree of correlation depended on the strength of the LD. Therefore, SNP pairs with similar allele frequencies were more frequent at very short distances because of higher LD. However, note that similar allele frequencies do not always imply high LD. This can be clearly seen in Figure 4. Therefore, usable LD among SNP measured by $r^{2}$ might not be substantially affected by MAF even for short distances. Small LD seems to be underestimated when the difference in $\mathrm{MAF}$ is large and the MAF of one locus is small. Contrary to the $D^{\prime}$ measure, $r^{2}$ is not inflated for SNP with rare alleles, but rather underestimated.

As additional information, the dependency of $D^{\prime}$ on MAF was also examined. The $D^{\prime}$ statistics generally gave inflated $\mathrm{LD}$, which is in accordance with findings in other studies (e.g., Du et al., 2007; Zhao et al., 2007). The $D^{\prime}$ values were more inflated for SNP pairs with a rare allele. Interestingly, mean $D^{\prime}$ values decreased as allele frequencies at both loci moved closer to 0.5 (data not shown).

Contrary to the previous studies that used $D^{\prime}$ as a measure of LD (Farnir et al., 2000; Odani et al., 2006), the current investigation did not find high levels of LD between unlinked markers. A very few nonsyntenic SNP pairs exhibited high LD $\left(r^{2}>0.3\right)$, which might be caused by selection, wrongly positioned SNP, or chance.

Based on analysis of covariance, significant differences in LD among the BTA $(P<0.0001)$ were found, which are in agreement with other published studies (Farnir et al., 2000; Tenesa et al., 2003; Odani et al., 2006). Selection is known to create interchromosomal LD heterogeneity, and because Holstein cattle have undergone intense selection during the past decades, this may be one of the causes of the estimated differences in LD across chromosomes in the current study. Selection can also cause LD between syntenic markers that harbor genes responsible for controlling one trait, which is known as the hitchhiking effect (e.g., Ardlie et al., 2002). Therefore, one may speculate whether the high LD between long distances observed on BTA 15, 20, and 21 was a consequence of genetic selection (Figure 3).

Linkage disequilibrium is not uniformly distributed throughout the bovine genome (e.g., Khatkar et al., 2007). Generally, the LD is expected to be small in regions of high recombination. Therefore, to assess local patterns of long-range $\mathrm{LD}$, genomic regions with low and high LD were identified by using the sliding window approach. The extent of LD could not be examined for nearly $55 \%$ of the autosomal genome when there were large gaps in the SNP map or when not enough SNP were located at a range of 0.2 to $0.6 \mathrm{Mb}$. In a few parts of the autosomal genome, discrete blocks of LD separated by possible recombination hot spots were observed. The regional pattern of $\mathrm{LD}$ is not expected to be noticeably affected by clusters of SNP located very close together, because the SNP spacing was partially accounted for by considering the SNP pairs spaced between 0.2 and $0.6 \mathrm{Mb}$. The results suggest that a denser SNP map will be required to provide fine assessment of LD blocks in the cattle genome, which is in agreement with reports by Spelman and Coppieters (2006) and Khatkar et al. (2007).

The $r^{2}$ is directly related to the statistical power in association studies. Pritchard and Przeworski (2001) showed that when using a marker to find an association with the nearby gene, the sample size should be increased by a factor of $1 / r^{2}$ to achieve the same power as using the causative gene directly. Accordingly, in several studies, the level of useful LD has been defined based on practical limits to increase in sample size (e.g., Kruglyak, 1999; Ardlie et al., 2002). In Holstein cattle, from a practical perspective, we assumed that a 3 -fold increase in sample size was the upper limit; therefore, roughly $r^{2}$ larger than 0.3 was considered as useful LD for mapping purposes. Based on the work of Meuwissen et al. (2001), the required level of $\operatorname{LD}\left(r^{2}\right)$ for genomic 
selection to achieve an accuracy of 0.85 for genomic breeding values (or a reliability of 0.72 ) seems to be 0.2 ( $r^{2}$ calculated based on the $N_{e}$ and genetic distance from their simulation study). Currently, there are no reports on the required level of $\mathrm{LD}$ for more accurate genomic selection; therefore, as a conservative criterion, it was also assumed that an $r^{2}>0.3$ would be useful for genomic selection. However, this threshold for useful LD is arguable and readers may decide to use another one.

Assuming that $r^{2}>0.3$ is useful for association studies and that the genes (or quantitative trait loci) of interest are located in the middle of 2 SNP (the worst situation with respect to $L D$ ), the extent of $L D$ observed in the current investigation indicates that the average resolution of the SNP map should be approximately less than $70 \mathrm{~kb}$ to capture a large amount of useful LD between SNP and the gene (assuming biallelic loci). This density is obtained by looking at the mean $r^{2}$ for close SNP pairs separated by different distances ( 0 to 5,5 to 10 , 10 to $15 \mathrm{~kb}$, and so on). Seventy-nine percent of SNP pairs separated by $\leq 35 \mathrm{~kb}$ showed an $r^{2}>0.30$. However, this is a rough average estimate, and the required resolution could easily be higher or lower at different regions across the genome because of the irregular distribution of recombination events along the chromosomes. A more accurate estimate of the required density can be obtained with a denser SNP map without large gaps. Once a reliable pattern of LD is available, denser SNP maps might be required in regions of low $\mathrm{LD}$, whereas sparse SNP maps might be sufficient for regions of high LD. In general, the required map resolution of $70 \mathrm{~kb}$ was much lower than that in humans (e.g., Dunning et al., 2000) because of the smaller effective population size in Holstein cattle.

A considerable number of pairs of SNP were spaced at $<10 \mathrm{~kb}$ distance and showed very high levels of LD. Tightly linked markers (usually in strong LD) might provide redundant information because they might be located on the same gene. The average gene size in cattle ranges from $\sim 37$ to $74 \mathrm{~kb}$, with very high variation, based on a cattle-human comparative map (Everts-van der Wind et al., 2005). Therefore, the mean LD between SNP located in different genes might be lower than what was found in the current study.

Because the extent of $\mathrm{LD}$ is affected by both recent and past $N_{e}$, estimating historical $N_{e}$ is useful to shed light on the evolutionary pattern of LD. In Holstein cattle in North America, the historical $N_{e}$, going back 500 generations, was found to be approximately 1,400 individuals, in contrast to the estimated $<100$ individuals in recent generations. Genomic regions with a low recombination rate were only partially accounted for because the linkage map for SNP was constructed based on a relatively loose genetic map of microsatellite mark- ers. Although the figures for $N_{e}$ might not be highly accurate, they nevertheless provide useful information on the trend in effective population size. In general, small $_{e}$ in recent generations in the Holstein population can be attributed to the intense selection of bulls combined with worldwide use of a small number of elite bulls. Goddard et al. (2006) observed similar trends for recent past $N_{e}$ in Holstein cattle in Australia, whereas Thevenon et al. (2007) reported considerably larger recent past $N_{e}$ for cattle populations of western Africa, which were not strongly selected.

\section{CONCLUSIONS}

Using a relatively dense SNP map (5,564 SNP), we found the LD (as measured by the $r^{2}$ statistic) to be low on average, to decay very rapidly within a few hundred kilobase pairs, and to show irregular patterns across the autosomal genome in Holstein cattle in North America. Results indicate that a denser SNP map is needed to capture the LD information required for whole-genome fine mapping and genomic selection and to completely assess the pattern of $\mathrm{LD}$ across the autosomal genome.

\section{ACKNOWLEDGMENTS}

The authors gratefully acknowledge financial support from L'Alliance Boviteq Inc. (St-Hyacinthe, Quebec, Canada) and the Ontario Centre for Agricultural Genomics, Guelph, Ontario, Canada (Challenge Fund). The contributions of Jacques Chesnais from Semex Alliance to this research are greatly appreciated.

\section{REFERENCES}

Affymetrix Inc. 2005. Affymetrix MegAllele GeneChip Bovine 10K SNP Array. Affymetrix Inc., South San Francisco, CA. http:// www.affymetrix.com/support/technical/datasheets/bovine10k snp_datasheet.pdf Accessed June 11, 2007.

Akey, J. M., K. Zhang, M. M. Xiong, P. Doris, and L. Jin. 2001. The effect that genotyping errors have on the robustness of common linkage-disequilibrium measures. Am. J. Hum. Genet. 68:1447-1456.

Ardlie, K. G., L. Kruglyak, and M. Seielstad. 2002. Patterns of linkage disequilibrium in the human genome. Nat. Rev. Genet. 3:299-309.

Du, F.-X., A. C. Clutter, and M. M. Lohuis. 2007. Characterizing linkage disequilibrium in pig populations. Int. J. Biol. Sci. $3: 166-178$

Dunning, A. M., F. Durocher, C. S. Healey, M. D. Teare, S. E. McBride, F. Carlomagno, C. F. Xu, E. Dawson, S. Rhodes, S. Ueda, E. Lai, R. N. Luben, E. J. Van Rensburg, A. Mannermaa, V. Kataja, G. Rennart, I. Dunham, I. Purvis, D. Easton, and B. A. J. Ponder. 2000. The extent of linkage disequilibrium in four populations with distinct demographic histories. Am. J. Hum. Genet. 67:1544-1554.

Everts-van der Wind, A., D. M. Larkin, C. A. Green, J. S. Elliott, C. A. Olmstead, R. Chiu, J. E. Schein, M. A. Marra, J. E. Womack, and H. A. Lewin. 2005. A high-resolution whole-genome cattlehuman comparative map reveals details of mammalian chromosome evolution. Proc. Natl. Acad. Sci. USA 102:18526-18531. 
Farnir, F., W. Coppieters, J. J. Arranz, P. Berzi, N. Cambisano, B. Grisart, L. Karim, F. Marcq, L. Moreau, M. Mni, C. Nezer, P. Simon, P. Vanmanshoven, D. Wagenaar, and M. Georges. 2000. Extensive genome-wide linkage disequilibrium in cattle. Genome Res. 10:220-227.

Gao, G., I. Hoeschele, P. Sorensen, and F.-X. Du. 2004. Conditional probability methods for haplotyping in pedigrees. Genetics 167:2055-2065.

George, A. W., P. M. Visscher, and C. S. Haley. 2000. Mapping quantitative trait loci in complex pedigrees: A two-step variance component approach. Genetics 156:2081-2092.

Goddard, M. E., B. Hayes, H. McPartlan, and A. J. Chamberlain. 2006. Can the same genetic markers be used in multiple breeds? CD-ROM Communication No. 22-16 in Proc. 8th World Congr. Genet. Appl. Livest. Prod., Belo Horizonte, Brazil.

Hayes, B. J., P. M. Visscher, H. C. McPartlan, and M. E. Goddard. 2003. Novel multilocus measure of linkage disequilibrium to estimate past effective population size. Genome Res. 13:635-643.

Hill, W. G., and A. Robertson. 1968. Linkage disequilibrium in finite populations. Theor. Appl. Genet. 38:226-231.

Khatkar, M. S., A. Collins, J. A. L. Cavanagh, R. Hawken, M. Hobbs, K. Zenger, W. Barris, A. E. McClintock, P. C. Thomson, F. W. Nicholas, and H. W. Raadsma. 2006. A first generation metric linkage disequilibrium map of bovine chromosome 6 . Genetics 174:79-85.

Khatkar, M. S., K. R. Zenger, M. Hobbs, R. J. Hawken, J. A. L. Cavanagh, W. Barris, A. E. McClintock, S. McClintock, P. C. Thomson, B. Tier, F. W. Nicholas, and H. W. Raadsma. 2007. A primary assembly of a bovine haplotype block map based on a $15 \mathrm{k}$ SNP panel genotyped in Holstein-Friesian cattle. Genetics 176:763-772.

Kruglyak, L. 1999. Prospects for whole-genome linkage disequilibrium mapping of common disease genes. Nat. Genet. 22:139-144.

Laan, M., and S. Paabo. 1997. Demographic history and linkage disequilibrium in human populations. Nat. Genet. 17:435-438.

Lange, K., and T. M. Goradia. 1987. An algorithm for automatic genotype elimination. Am. J. Hum. Genet. 40:250-256.

Lewontin, R. C. 1964. The interaction of selection and linkage. I. General considerations; heterotic models. Genetics 49:49-67.

Lewontin, R. C. 1988. On measures of gametic disequilibrium. Genetics 120:849-852.

McRae, A. F., J. C. McEwan, K. G. Dodds, T. Wilson, A. M. Crawford, and J. Slate. 2002. Linkage disequilibrium in domestic sheep. Genetics 160:1113-1122.

Meuwissen, T. H. E., and M. E. Goddard. 2000. Fine mapping of quantitative trait loci using linkage disequilibria with closely linked marker loci. Genetics 155:421-430.

Meuwissen, T. H. E., B. J. Hayes, and M. E. Goddard. 2001. Prediction of total genetic value using genome wide dense marker maps. Genetics 157:1819-1829.

National Center for Biotechnology Information. 2007. Bovine Microsatellite Framework cM Map. NCBI Online. http://www.ncbi. nlm.nih.gov/mapview/ Accessed Mar. 5, 2007.

Odani, M., A. Narita, T. Watanabe, K. Yokouchi, Y. Sugimoto, T. Fujita, T. Oguni, M. Matsumoto, and Y. Sasaki. 2006. Genomewide linkage disequilibrium in two Japanese beef cattle breeds. Anim. Genet. 37:139-144.

Pritchard, J. K., and M. Przeworski. 2001. Linkage disequilibrium in humans: Models and data. Am. J. Hum. Genet. 69:1-14.

Risch, N., and K. Merikangas. 1996. The future of genetic studies of complex human diseases. Science 273:1516-1517.

Sandor, C., F. Farnir, S. Hansoul, W. Coppieters, T. Meuwissen, and M. Georges. 2006. Linkage disequilibrium on the bovine X chromosome: Characterization and use in quantitative trait locus mapping. Genetics 173:1777-1786.

Spelman, R. J., and W. Coppieters. 2006. Linkage disequilibrium in the New Zealand Jersey population. CD-ROM Communication No. 22-21 in Proc. 8th World Congr. Genet. Appl. Livest. Prod., Belo Horizonte, Brazil.

Sved, J. A. 1971. Linkage disequilibrium and homozygosity of chromosome segments in finite populations. Theor. Pop. Biol. 2:125-141.
Tenesa, A., S. A. Knott, D. Ward, D. Smith, J. L. Williams, and P. M. Visscher. 2003. Estimation of linkage disequilibrium in a sample of the United Kingdom dairy cattle population using unphased genotypes. J. Anim. Sci. 81:617-623.

Thevenon, S., G. K. Dayo, S. Sylla, I. Sidibe, D. Berthier, H. Legros, D. Boichard, A. Eggen, and M. Gautier. 2007. The extent of linkage disequilibrium in a large cattle population of western Africa and its consequences for association studies. Anim. Genet. 38:277286.

Uimari, P., O. Kontkanen, P. M. Visscher, M. Pirskanen, R. Fuentes, and J. T. Salonen. 2005. Genome-wide linkage disequilibrium from 100,000 SNPs in the East Finland founder population. Twin Res. Hum. Genet. 8:185-197.

Yamazaki, T. 1977. The effects of overdominance on linkage in a multilocus system. Genetics 86:227-236.

Zhao, H., D. Nettleton, and J. C. M. Dekkers. 2007. Evaluation of linkage disequilibrium measures between multi-allelic markers as predictors of linkage disequilibrium between single nucleotide polymorphisms. Genet. Res. 89:1-6.

Zhao, H., D. Nettleton, M. Soller, and J. C. M. Dekkers. 2005. Evaluation of linkage disequilibrium measures between multi-allelic markers as predictors of linkage disequilibrium between markers and QTL. Genet. Res. 86:77-87.

Zhao, H., R. Pfeiffer, and M. H. Gail. 2003. Haplotype analysis in population genetics and association studies. Pharmacogenomics 4:171-178.

\section{APPENDIX 1}

\section{Haplotype Reconstruction Algorithm}

Initialization. Let subscript $i$ denote animal $i$ and subscript $j$ denote marker $j$. Set $P_{i j}$ to 1 or 0 for animals that are homozygous for allele 1 or 2 , respectively, or for heterozygous animals whose sire is homozygous for allele 1 or 2, respectively. For other heterozygous animals, set $P_{i j}$ to 0.5 . Finally, for animals whose sire has not been genotyped, set $P_{i j}$ to 1 at the first heterozygous marker on each chromosome.

Step 1. Adjust $P_{i j}$ by using progeny information:

1. Process sires from youngest to oldest.

2. For each heterozygous marker $(j)$ with an uncertain phase $\left(0<P_{i j}<1\right)$,

a. find the nearest partially informative heterozygous marker $\left(j^{\prime}\right)$; that is, $\left|P_{i j^{\prime}}-0.5\right|>K_{1}$, where $K_{1}$ is a threshold value for partial informativeness of a heterozygous marker,

b. compute an effective number of nonrecombinant progeny, $z$, given that the sire's paternal allele is 1 at $j$, and $j^{\prime}$ :

$$
z=\Sigma_{k}\left(2\left(P_{k j}+P_{k j^{\prime}}-2 P_{k j} P_{k j^{\prime}}\right)-1\right), \text { and }
$$

c. compute an updated $P_{i j}$ as

$q\left(1-P_{i j}\right)+(1-q) P_{i j}$,

where $q=\frac{(1-\theta)^{z}}{\theta^{z}+(1-\theta)^{z}}$ and $\theta$ is the recombination rate between $j$ and $j^{\prime}$. 
Step 2. Adjust $P_{i j}$ by using own and sire information at the flanking markers:

1. Process sires from youngest to oldest.

2. Process each marker $(j)$ with an uncertain phase for son $(i)$ of sire $(s)$ :

a. find the nearest partially informative marker on the left (L), that is, heterozygous sire and $\left|X_{L}-0.5\right|>K_{2}$, where $X_{L}=P_{i L} P_{s L}+\left(1-P_{i L}\right)(1$ $\left.-P_{s L}\right)$ and $K_{2}$ is a threshold value for partial informativeness of a heterozygous marker;

b. similarly, find the nearest partially informative marker on the right $(\mathrm{R})$;

c. let $\theta L\left(\theta_{R}\right)$ be the recombination rates between the left (right) flanking marker and marker $j$, and let $\theta$ be the recombination rate between the 2 flanking markers;

d. if there are suitable markers to the left and right, compute

$$
\begin{aligned}
& X=X_{L} X_{R}\left(\frac{\left(1-\theta_{L}\right)\left(1-\theta_{R}\right)}{1-\theta}\right)+X_{L}\left(1-X_{R}\right)\left(\frac{\left(1-\theta_{L}\right) \theta_{R}}{\theta}\right) \\
& +\left(1-X_{L}\right) X_{R}\left(\frac{\theta_{L}\left(1-\theta_{R}\right)}{\theta}\right)+\left(1-X_{L}\right)\left(1-X_{R}\right)\left(\frac{\theta_{L} \theta_{R}}{1-\theta}\right)
\end{aligned}
$$

else if there is no suitable marker on the right or left, compute

$$
\begin{aligned}
& X=X_{L}\left(1-\theta_{L}\right)+\left(1-X_{L}\right) \theta_{L} \text { or } \\
& X=X_{R}\left(1-\theta_{R}\right)+\left(1-X_{R}\right) \theta_{R}, \text { respectively; and }
\end{aligned}
$$

e. compute an updated $P_{i j}$ as $P_{i j}+(q-0.5)(1-$ $\left.\left|P_{i j}-0.5\right|\right)$, where $\mathrm{q}=P_{s j} X+\left(1-P_{s j}\right)(1-\mathrm{X})$.

Iterate. Repeat steps 1 and 2 until the sum of squared changes in $P_{i j}$ is sufficiently small. Finally, the paternal allele at each heterozygous locus is set to 1 for $P_{i j} \leq 0.5$ and to 2 for $P_{i j}>0.5$. In the simulated data sets described in the main text, the best results were found by using $K_{1}=K_{2}=0.1$ and performing 8 to 10 iterations. 